\title{
LAPIV using multi-resolution warping and proxy regularization
}

\author{
Yin Yang ${ }^{1 *}$, Dominique Heitz ${ }^{1}$ \\ ${ }^{1}$ INRAE, UR OPAALE, F-35044 Rennes Cedex, France \\ * correspondent author: yin.yang@inrae.fr
}

\section{Abstract}

The Lagrangian Particle Image Velocimetry (LAPIV) method was firstly proposed in Yang et al. (2019) as a prototype approach to achieve the goal of accurate and efficient reconstruction of 3D Eulerian velocity field of fluid flow from multi-view particle images. After validating against synthetic datasets, the prototype has already shown significant advantages in revealing more small scale flow structures than other stateof-the-art Eulerian velocity estimation methods, such as TomoPIV (Scarano, 2013) and VIC\# (Jeon et al., 2019). However, at this early stage, LAPIV can not be easily applied to other datasets. In the current work, we focus on extending LAPIV to operational search by incorporating several essential and wellestablished paradigms: multi-resolution, warping, and proxy regularization. Recent approaches, Lasinger et al. (2019) and Cornic et al. (2020), function in the same vein as LAPIV, aiming at reconstructs the dense Eulerian volumetric flow directly from multi-view particle-seeded images Another pipeline consists of firstly reconstructing the Lagrangian flow using the Lagrangian Particle Tracking (LPT), then optimally interpolating the Lagrangian flow to Eulerian grids, taking into account the Eulerian dynamics constraints as in Flowfit (Gesemann, 2020) and VIC\# (Jeon et al., 2019). If Eulerian flow is required, LAPIV is the preferred approach due to its simplicity and ability to utilize the original rich image features.

LAPIV is built on the two pillars of local quasi-rigidity assumption and kernel method. For two-pulse double frame data, LAPIV functions as follows: 1) Reconstruct the particle field (position and intensity) of the first frame using IPR (Wieneke, 2012); 2) Prescribe the fixed Eulerian grid with a resolution depending on the particle density. 3) For each grid point, find all particles in its neighborhood and assume those particles follows the same motion as the grid point; 4) Solve for the velocity at the grid point by minimizing a cost function measuring the discrepancy in image space between the recorded image at the second frame and the projected image produced by an image model (Transport equation + Camera model + Optical transfer function (OTF)); 5) Post-process. Step 3 consists of finding particles in the 'support region' near the centering grid. We employ the word 'support' to denote that the transportation of the particles in the region can be contributed by the centering velocity. Step 4 involves the kernel principle (Yang and Heitz (2021)). Despite the nonlinear relationship between the projected image pixel intensity and the velocity at a given grid point, by finding a kernel function that can effectively match the projected particle image with the recorded image, we can train a function that maps the image features to the velocity from few generated samples. Then the learned function can be applied on the recorded image to infer the real velocity.

Nevertheless, at the prototyping stage, the above algorithm has several drawbacks. First, convergence and precision of the algorithm can hardly be achieved simultaneously with one layer Eulerian grid. Estimated velocity on a coarser grid tends to converge better but at the risk of losing small scale information. On the other hand, working on a finer grid generally leads to lower local data discrepancy but not always more accurate velocity as wrong particle pairs are more likely to be matched. Our strategy is to implement the multi-resolution technique and warping. We start the estimation at a coarser grid and gradually refine the estimation on a finer grid. The estimation process on the finer grid begins by inheriting an initial velocity field from the previous iteration on the coarse grid through warping instead of using zeros. Second, holes are commonly presented in final velocity estimation resulting from a lack of particles at certain spots. Inhomogeneous seeding is inevitable for real experiments. As a result, some interpolation schemes must be employed to fill velocity holes with meaningful values based on their neighbors. Rather than treating the interpolation scheme naively in the post-processing step in the prototype stage, we formalize the optimal interpolation under variational settings using proxy regularization. We integrate a flow regularization step right after the proxy flow estimation is obtained at each grid level. Here we use the term 'proxy' to denote the flow estimated from step 4 (kernel methods) of LAPIV. The regularized flow respects various 
physical constraints and resembles the proxy flow as closely as possible. The resulting system to solve for the regularized flow remains light-weight compared to Lasinger et al. (2019), or Gesemann (2020), whose optimization methods are quite complex since they anchor either the image or the particle field as the data. As for LAPIV, the regularized flow always occupies the same grid as the proxy flow. Both are to be refined in agreement with the multi-resolution approach.

We have participated in the 1st LPT challenge after a slight modification of the original algorithm, as the challenge emphasized LPT rather than the Eulerian velocity reconstruction. As evaluated by the challenge, our method has achieved state-of-the-art performance at medium-high particle density levels both for twopulse datasets (PPP $<=0.08)$ and time-resolve datasets (PPP $<=0.12$ ). We have also done quantitative tests against the same synthetic datasets depicting the wake flow of a turbulent cylinder at $\mathrm{Re}=3900$, produced by a Large Eddy Simulation (Parnaudeau et al., 2008). Table 1 summarizes the RMSE results of LAPIV compared to TomoPIV, STB-bin and VIC\# implemented in Davis 10.0.5. LAPIV yielded the minimum error among all methods. Finally, we have applied LAPIV to an impinging jet flow Yang and Heitz (2021) at $\operatorname{Re}=1250$. The $\mathrm{Q}$ criteria of the instantaneous velocity field are shown in figure 1 with color-coded by the velocity magnitude. In this study, we have shown, after integrating those essential techniques such as multi-resolution and proxy regularization, LAPIV can yield competitive, if not better, results compared to other state-of-the-art approaches facing versatile datasets.

\begin{tabular}{c|c|c} 
& & \\
Methods & $\begin{array}{c}\text { Resolution } \\
\left(N_{x}, N_{y}, N_{z}\right)\end{array}$ & $\begin{array}{c}\text { RMSE } \\
\left(U_{\text {err }} / U_{\infty}\right)\end{array}$ \\
\hline TomoPIV & $(145,45,24)$ & 0.1517 \\
\hline STB-bin & $(145,45,24)$ & 0.2439 \\
\hline VIC\# & $(145,45,24)$ & 0.09141 \\
\hline LAPIV & $(151,48,27)$ & 0.07799 \\
\hline
\end{tabular}

Table 1: Summary of results obtained by TomoPIV, STB binning, VIC\# (all three methods are implemented in Davis 10.0.5) and LAPIV for datasets of wake flow behind cylinder at $\mathrm{Re}=3900$.

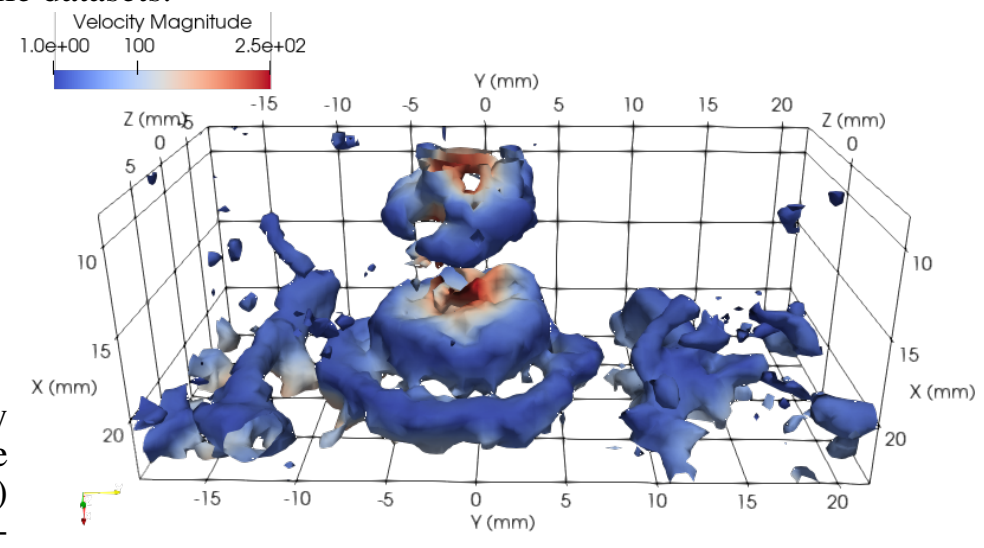

Figure 1: An isosurface of the $\mathrm{Q}$ criteria criteria in an impinging jet flow at $\operatorname{Re}=1250$, color coded by the velocity magnitude $(\mathrm{mm} / \mathrm{s})$.

\section{References}

Cornic P, Leclaire B, Champagnat F, Le Besnerais G, Cheminet A, Illoul C, and Losfeld G (2020) Doubleframe tomographic ptv at high seeding densities. Experiments in Fluids

Gesemann S (2020) Flowfit3: Fast data assimilation for recovering instantaneous details of incompressible flows based on scattered data. in 3rd Workshop and 1st Challenge on DA \& CFD for PIV and LPT

Jeon YJ, Müller M, Michaelis D, and Wieneke B (2019) Data assimilation-based flow field reconstruction from particle tracks over multiple time steps. in 13th ISPIV

Lasinger K, Vogel C, Pock T, and Schindler K (2019) 3D Fluid Flow Estimation with Integrated Particle Reconstruction. International Journal of Computer Vision

Parnaudeau P, Carlier J, Heitz D, and Lamballais E (2008) Experimental and numerical studies of the flow over a circular cylinder at reynolds number 3900. Physics of Fluids

Scarano F (2013) Tomographic PIV: principles and practice. Measurement Science and Technology

Wieneke B (2012) Iterative reconstruction of volumetric particle distribution. Measurement Science and Technology

Yang Y and Heitz D (2021) Kernelized lagrangian particle tracking. hal-03212696

Yang Y, Heitz D, and Mémin E (2019) Lagrangian particle image velocimetry. in 13th ISPIV 